\title{
Morphological changes in the skin microlymphatics in recently injured paraplegic patients with ilio-femoral venous thrombosis
}

\author{
R Scelsi ${ }^{1}$, L Scelsi ${ }^{1}, \mathrm{R}$ Bocchi $^{2}$ and S Lotta ${ }^{2}$ \\ ${ }^{1}$ Department of Human Pathology, University of Pavia, Via Forlanini 14, 27100 Pavia; ${ }^{2}$ G Verdi Rehabilitation \\ Center of Villanova d'Arda, USL 3, Villanova d'Arda, Italy
}

Based on morphological features of the lymphatic microcirculation of the skin from healthy subjects, and from paraplegic patients who had no evidence of ilio-femoral venous thrombosis (thromboembolic disease: TED), the leg terminal lymphatic vessels from skin biopsies of five male paraplegic patients with acute traumatic spinal cord lesions and with documented TED were studied. Paraplegic patients with TED had lymph vessels with a dilated lumen surrounded by a rarefacted perivascular connective tissue characterized by dissociation and disruption of collagen and elastic fibres. The lymphatic wall was generally attenuated and some open junctions and channels delimited by endothelial protrusions were observed. The venous outflow obstruction caused by deep venous thrombosis accompanied by the absence of ambulatory venous pressure in the paretic leg determines skin microlymphatic dilatation, lymph stasis and changes in the interstitial connective tissues. These alterations may be considered to be the morphological aspect of the dystrophic alterations seen in the skin of legs from paraplegic patients with TED. The results are discussed in view of the correct rehabilitative medical treatment necessary, and adequate prophylaxis of TED.

Keywords: thromboembolic disease; paraplegia; lymph vessels; morphology

\section{Introduction}

Thromboembolic disease (TED) is an important cause of morbidity and mortality in paraplegic patients during the first 2 to 3 months after spinal cord injury. ${ }^{1}$ Its aetiology includes venous stasis, prolonged bed rest, immobilisation and loss of muscle contraction.

The incidence of clinically apparent TED in acute paraplegic patients without chemical prophylaxis has been reported to range from $12.5 \%$ to $40 \% .^{2,3}$ After adequate pharmacological and mechanical prophylaxis for TED, the incidence of the thromboembolic complications was greatly reduced. ${ }^{1-5}$ In paraplegic subjects, the increased deep venous resistance and the venous stasis determines microangiopathic changes in the skin of the lower extremities involving lymphatic vessels and leading to skin alterations of variable extent with oedema.

Recent microlymphographic and morphological investigations on walking patients with TED demonstrated microangiopathic alterations in the skin inducing dystrophic changes with oedema. ${ }^{6,7}$ Considering these studies, we have tried to perform a morphological investigation on the lymphatic microcirculation of the skin of paraplegic patients with TED.

In the following study, we will report on the light and electron microscopic features of lymphatic vessels seen in skin biopsies of the lower extremities from paraplegic patients with TED, in order to assess the possible role of microlymphatic alterations in the development of dystrophic skin alterations. Skin microlymphatic morphological aspects were compared to those seen in either normal healthy subjects, or in paraplegic patients with no evidence of TED.

\section{Patients and methods}

A total of five male paraplegic patients with an acute spinal cord lesion (lesion level T1-10) and with documented ilio-femoral venous thrombosis were studied. The patients were admitted to the G. Verdi Rehabilitative Centre in Villanova d'Arda during 1993 and 1994. The protocol of this study was approved by the regional review board and the patients provided informed written consent for the needle skin biopsy.

The ages of the patients were 17, 18, 21, 28 and 33 years, respectively. All five cases of TED first became symptomatic from 2 to 11 weeks after the acute spinal cord injury.

The paraplegic patients were nursed in a standard way, and there was no difference in the pressure care that they received (water cushions).

The clinical signs included spastic paraplegia, asymmetrical swelling and warmth of the legs, engorged superficial veins, oedema and dystrophic changes of the skin of the lower extremities. Confirmatory studies such as plethysmography, venography, venous doppler and 125 I-labeled fibrinogen were performed.

Skin microlymphatic morphological aspects were 
compared to those seen in five paraplegic patients (mean age 24 years) with no evidence of TED and in two normal healthy subjects in which the skin biopsy was performed during orthopaedic surgery.

Needle skin biopsies were taken under local anaesthesia from the lateral aspect of the leg in which initial dystrophic skin alterations were seen. The specimens were fixed in a mixture of glutaraldehyde $(2.5 \%)$ and paraformaldehyde $(2 \%)$ in a sodium cacodylate buffer $0.1 \mathrm{M}\left(\mathrm{pH} \mathrm{7.4)}\right.$ for $4 \mathrm{~h}$ at $+4{ }^{\circ} \mathrm{C}$. The samples were postfixed in $1 \%$ Os04 in $0.2 \mathrm{M}$ collidine buffer ( $\mathrm{pH} 7.4$ ) and were then dehydrated and embedded in epoxy resin.

Semithin sections $(0.5 \mu \mathrm{m})$ stained with toluidine blue were observed and recorded at the light microscope. Ultrathin sections $(40-80 \mathrm{~nm})$ were contrasted with uranil acetate and lead citrate and were observed at the electron microscope Zeiss EM10.

Some ultrathin sections were contrasted with orcein in aqueous solution $(2 \%)$ to detect the elastic fibres.

\section{Results}

Skin biopsies from normal healthy subjects

With the light microscope the dermal blood and lymphatic microcirculation of the skin from normal ambulant subjects showed a normal appearance. With electron microscopy investigation, the lymphatics showed a narrow lumen delimited by a thin and indented wall showing some open junctions and rare intercellular channels. The surrounding connective perivascular matrix was characterized by regular bundles of collagen and elastic fibres (Figure 1). The external side of endothelial cell line was joined to the surrounding connective tissue by thin anchoring filaments.

\section{Skin biopsies from paraplegic patients with no evidence of TED}

With the light microscope, lymphatic vessels showed a dilated lumen delimited by thin endothelial cells surrounded by a dense connective perivascular tissue. Ultrastructural investigation revealed a thin and flattened endothelial wall presenting rare open junctions and intercellular channels. Anchoring extravascular filaments with normal ultrastructural appearance were sometimes joined to the endothelial cells. No rarefaction or dissociation of collagen and elastic fibres in the perivascular tissue was observed.

\section{Skin biopsies from paraplegic patients with evidence of TED}

The terminal lymphatics of paraplegic patients with TED were easily recognizable because of their very dilated lumen surrounded by a rarefacted perivascular connective matrix characterized by dissociation and disruption of collagen and elastic fibres (Figure 2). The lymphatic endothelial wall was generally attenuated and indented. Numerous open junctions along the endothelial cells were observed. These elements were

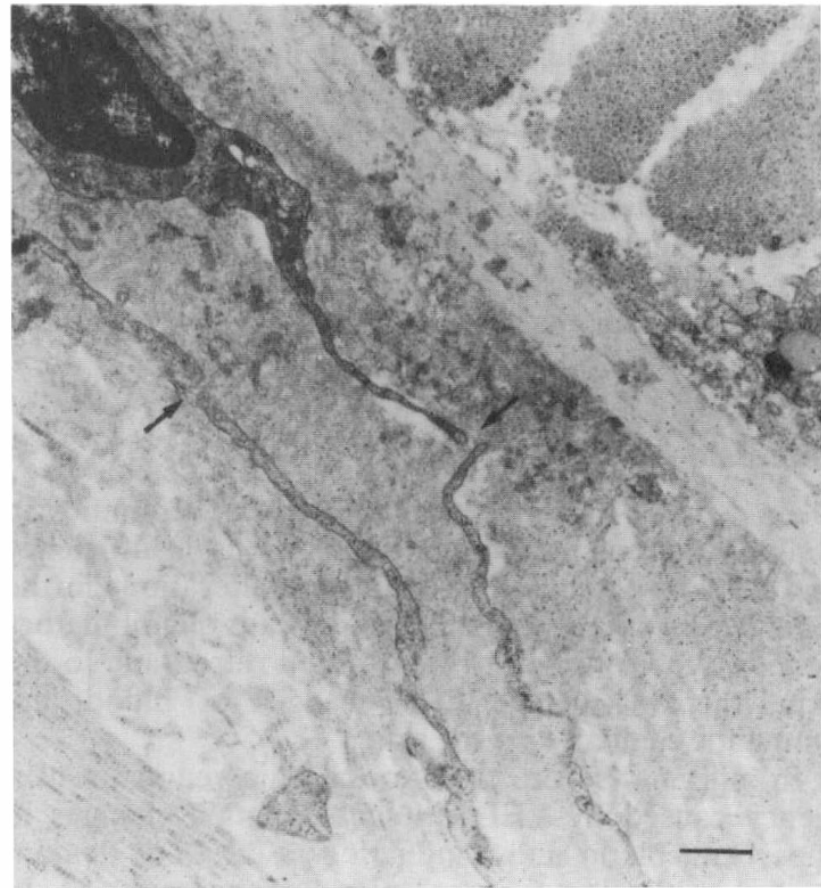

Figure 1 A terminal lymphatic from the normal skin showing a thin endothelial wall, some open junctions (arrows) and perivascular elastic and collagen fibres arranged in packed groups. (Electron microscopy; $\times 4,000$. Bar $=2 \mu$ )

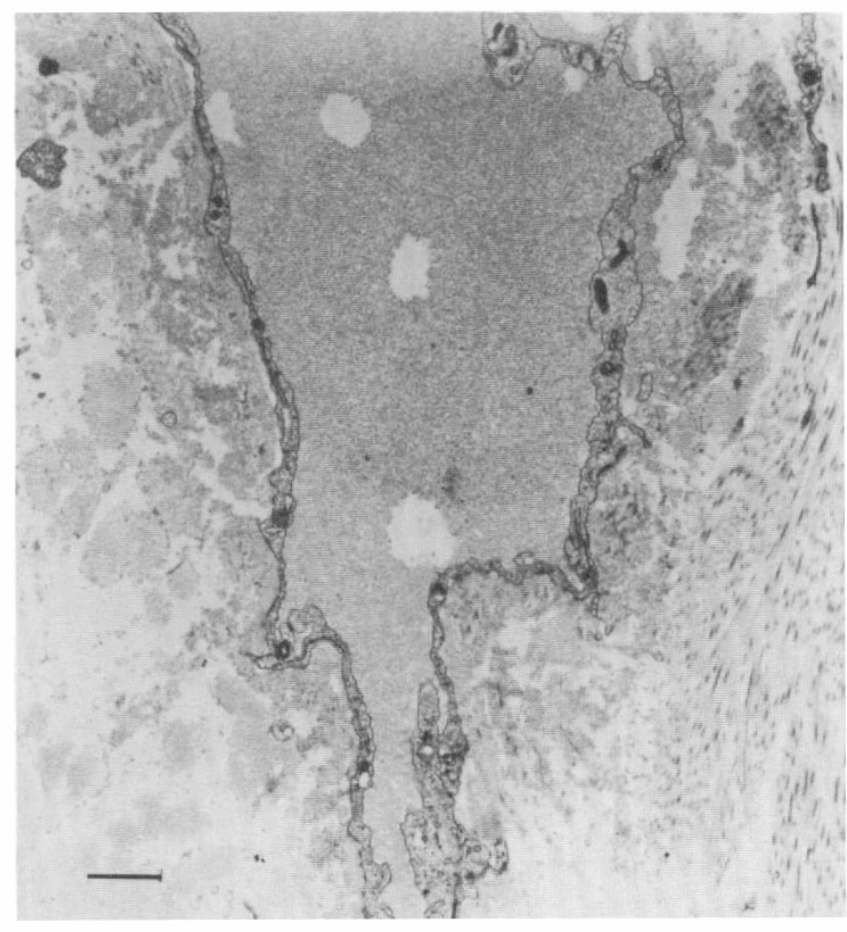

Figure 2 A terminal lymphatic from a paraplegic patient with TED showing a dilated lumen filled by lymph and numerous micropynocitotic vescicles. The perivascular connective tissue is disorganized by oedema (Electron microscopy; $\times 4,000$. Bar $=2 \mu$ ) 
characterized by dark and indented nuclei and by cytoplasmic vacuolisation. In some instances, endothelial cytoplasmic protrusions delimited channels in which granular material was present (Figure 3).

The connective tissue strictly lying on the abluminal side of the lymphatic endothelium showed vacuoles filled by fine dense material. Collagen and elastic fibres were dissociated and sometimes disrupted, but the anchoring filaments were normally present near the endothelial wall.

\section{Discussion}

Deep venous thrombosis of the lower extremities in walking patients causes chronic venous insufficiency and leads eventually to trophic skin alterations in the legs. During walking, the pressure heads of ambulatory venous hypertension are transmitted retrograde to the skin microvasculature with changes of blood and lymphatic capillaries of the skin.

In mild and severe states of chronic venous insufficiency caused by TED microangiopathic changes in superficial blood and lymphatic vessels were demonstrated with fluorescence microlymphography. ${ }^{6,8}$ In these conditions the morphological study of skin biopsies showed collapsed terminal lymphatics delimited by endothelial cells strictly connected with complex interdigitations, and scanty open junctions and intercellular channels. ${ }^{7,9}$

These morphologic alterations appear to be respons-

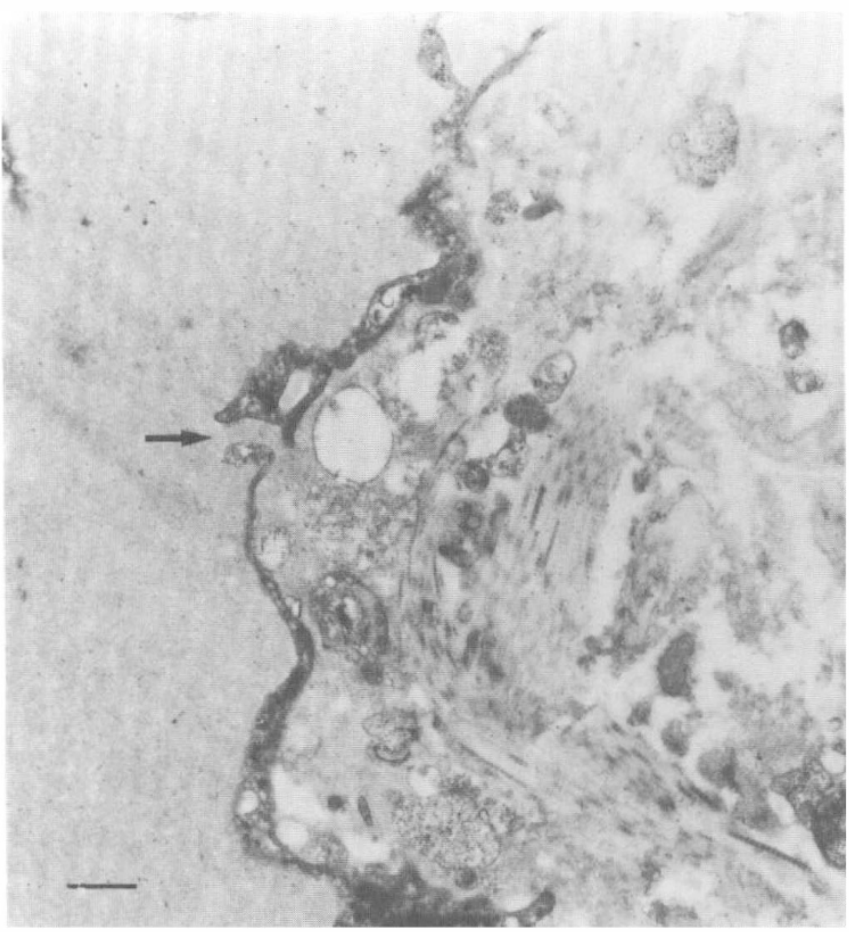

Figure 3 A lymphatic vessel from a paraplegic patient with TED is characterized by an open junction between endothelial cells (arrow) and by free granular material and vacuoles between disorganized elastic and collagen fibres. (Electron microscopy; $\times 12,000 . \mathrm{Bar}=1 \mu$ ) ible for an impairment of interstitial fluid exchanges in ambulatory patients with TED.

Recent studies demonstrated that the venous vascular properties in the legs of paraplegic patients with no evidence of TED have changed, ie a decrease in venous distensibility and capacity, and an increase in venous flow resistance. ${ }^{10}$ In these patients lymph vessels showed a dilated lumen indicating initial functional alterations probably related to immobilisation and bedrest, but no evidence of oedema or of changes in the perivascular connective tissues was seen. ${ }^{11}$ The described venous and lymphatic alterations are most probably the result of vascular adaptations to inactivity and muscle atrophy. ${ }^{10,11}$

In those with paraplegia, thromboembolic disease depends on various conditions such as loss of skeletal muscle contraction, immobilisation and venous stasis. In paraplegic patients with TED, dermal complications were present and they were characterized by dilatation of lymphatic microvessels and by changes in the interstitial connective tissues. The venous outflow obstruction caused by deep venous thrombosis accompanied by the absence of ambulatory venous pressure in the paretic leg determines the described skin microlymphatic dilatation with consequent endoluminal lymph stasis and increased transcapillary diffusion of the lymph material into the interstitial perivascular connective tissues, with reduced removal of tissue catabolites. These alterations may be considered the morphological aspect of a microlymphatic disease that, together with alterations of the blood capillaries combine to determine dystrophic changes in the skin of the legs from paraplegic patients with TED.

Adequate prophylaxis with anticoagulation therapy and a correct rehabilitative medical treatment from the occurrence of the spinal cord lesion diminishes some risk factors intervening in the aetiology of TED.

\section{References}

1 Perkash A. Experience with the management of thromboembolism in patients with spinal cord injury. Part I. Incidence, diagnosis and role of some risk factors. Paraplegia 1978; 16: 322-331.

2 Watson $\mathrm{N}$. Venous thrombosis and pulmonary embolism in acute spinal cord injury. Paraplegia 1968; 6: 113-121.

3 Van Hove E. Prevention of thrombophlebitis in spinal cord injured patients. Paraplegia 1978; 16: 322-325.

4 Merli GJ et al. Mechanical plus pharmacological prophylaxis for deep vein thrombosis in acute spinal cord injury. Paraplegia 1992; 30: $558-562$

5 Kulkarpi JR, Burt AA, Tromans AT, Constable PDI. Prophylactic low dose heparin anticoagulant therapy in patients with spinal cord injuries: a retrospective study. Paraplegia 1992; 30: $169-172$

6 Franzeck UK, Haselbach P, Speiser D, Bollinger A. Microangiopathy of cutaneous blood and lymphatic capillaries in chronic venous insufficiency. The Yale J Biol Med 1993; 66: $37-46$.

7 Scelsi R, Scelsi L, Cortinovis R, Poggi P. Morphological changes of dermal blood and lymphatic vessels in chronic venous insufficiency of the leg. Int Angiology 1994; 13: 26-31.

8 Bollinger A, Pfister G, Hoffman U, Franzeck UK. Fluorescence microlymphography in chronic venous incompetence. Int Angiology 1989; 8 (suppl 4): 23-26. 
9 Wenner A, Leu HJ, Brunner U. Ultrastructural changes of capillaries in chronic venous insufficiency. Exp Cell Biol 1980; 48: $1-14$.

10 Hopman MTE et al. Properties of the venous vascular system in the lower extremities of individuals with paraplegia. Paraplegia
1994; 32: 810-816.

11 Scelsi R, Scelsi L, Poggi P. Microlymphatic changes in acute spinal cord injury. A morphological study on skin biopsies. VI Meeting of European Group of Lymphology (GEL), Bruxelles. 8 May 1994 (abstract 12). 